\title{
TÉCNICA FACTORIAL Y ANÁLISIS TERRITORIAL: PROPUESTA DE CONFIGURACIÓN DE LOS GRUPOS DE ACCIÓN LOCAL EN EXTREMADURA ${ }^{1}$
}

\author{
A. Pérez Díaz y F. Leco Berrocal \\ Grupo de Estudios sobre Desarrollo Rural y Local (GEDERUL) \\ Instituto de Investigación en Patrimonio (i-PAT) \\ Universidad de Extremadura \\ Campus Universitario, 10071 Cáceres \\ aperez@unex.es; fleco@unex.es
}

\begin{abstract}
Resumen: Lo Grupos de Acción Local extremeños manifiestan marcados contrastes en su extensión, volumen poblacional, densidad, número de municipios y caracterización territorial. El objetivo de este artículo es valorar el grado de idoneidad alcanzado en la implementación de las políticas de desarrollo rural y, con ello, sopesar la pertinencia o no de realizar ajustes territoriales en todos o en algunos de los GAL regionales.

La metodología aplicada ha consistido en un estudio de la situación de los veinticuatro Grupos de Acción Local según los resultados del Programa Operativo 2007-2013. Posteriormente, se ha aplicado la técnica del análisis multifactorial, que ha permitido la interpretación de esa compleja realidad y realizar diversas propuestas de ajuste territorial que mejorarían sensiblemente los niveles de eficacia y eficiencia en la gestión y aplicación de los fondos comunitarios getionados por los Grupos de Acción Local.
\end{abstract}

Palabras clave: desarrollo rural, LEADER, Grupo de Acción Local (GAL), análisis factorial.

Recibido: 15-09-2017. Aceptado: 15-11-2017.

1. El presente trabajo fue financiado por la Consejería de Agricultura, Desarrollo Rural, Medio Ambiente y Energía de la Junta de Extremadura, bajo el título "Estudio de la configuración y características de los GAL de Extremadura y de la normativa para la aplicación del PDR 2014-2020 y elaboración de propuesta más adecuada de configuración de los GAL para dicho período". Contrato Arto 83 LOU. Ref. UEx 184/14. 


\begin{abstract}
The Local Action Groups in Extremadura (Spain) show marked contrasts in their size, population size, density, number of municipalities and territorial characterization. The objective of this paper is to assess the degree of adequacy achieved in the implementation of rural development policies and, therefore, to consider the relevance or not of making territorial adjustments in all or some of the groups. The applied methodology has consisted in a study of the situation of the twenty-four Local Action Groups according to the results of the Operational Program 2007-2013. Subsequently, the technique of multifactorial analysis has been applied, which has allowed the interpretation of this complex reality and make various proposals for territorial adjustment that would significantly improve the levels of efficiency and efficiency in the management and application of community funds managed by the Groups of Local Action.
\end{abstract}

Keywords: rural development, LEADER, Local Action Group (LAG), factorial analysis.

\title{
1. Introducción
}

Localizada en el cuadrante suroccidental de la Península Ibérica, Extremadura constituye una de las diecisiete Comunidades Autónomas del Estado español. Se en-

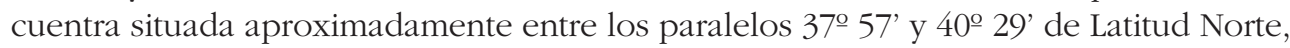
y los meridianos 4o 39' y 7ㅇ 33' de Longitud Oeste (según meridiano de Greenwich). Tiene una extensión de 41.634,5 $\mathrm{Km}^{2}$ y, a 1 de enero de 2016 (INE, 2017), contabiliza una población total de 1.087.778 habitantes que se reparten en un total de dos provincias, Badajoz y Cáceres, y 387 municipios. De ellos, sólo catorce superan el umbral de los 10.000 habitantes, en tanto que un $75 \%$ se encuentra por debajo de los 2.000.

En el territorio extremeño confluyen algunos de los rasgos más definitorios del mundo rural europeo, en general, y español, en particular: baja densidad de población (26,1 habitantes $/ \mathrm{Km}^{2}$, frente a una media nacional de 92 habitantes $/ \mathrm{Km}^{2}$ ), dispersión geográfica de la población, dependencia agraria de la economía (6 \% del PIB, casi tres veces superior a la media nacional), escaso desarrollo de las actividades industriales $(8,3$ $\%$ de la población activa), baja renta per cápita, debilidad económica, (es la única Comunidad Autónoma española considerada como región convergencia), fuerte incidencia del paro $(28,31 \%$ en el cuarto trimestre de 2016, la tasa más elevada del país, frente a una media nacional del 18,63 \%), elevado grado de envejecimiento demográfico (141,6 mayores de 65 años por cada 100 jóvenes, frente a una media nacional del 120,1\%), proceso de masculinización en edades fértiles, quiebra del crecimiento natural $(-1,4 \% 0)$ y persistencia de la emigración como una de las escasas alternativas de que dispone la población joven para alcanzar sus objetivos socioeconómicos (VV.AA., 2014). 
En virtud de estos condicionamientos, Extremadura constituye un excelente laboratorio para analizar el impacto de las políticas de Desarrollo Rural (Leco et al., 2017; Nieto y Cárdenas, 2015 y 2016; Esparcia y Escribano, 2012) y, sobre todo, como es el objetivo del presente trabajo, para evaluar el grado de eficacia y eficiencia con que éstas se han aplicado por parte de los 24 Grupos de Acción Local (en adelante, GAL) existentes en la región durante el período de Programación 2007-2013.

A este respecto, debe significarse que Extremadura se incorporó prontamente a la Iniciativa Comunitaria LEADER. En la primera convocatoria realizada en 1991, lo hicieron las comarcas de Sierra de Gata, Valle del Jerte, Alcántara y La Serena. El éxito alcanzado dio lugar a que, en 1995, fueran 10 los Grupos de Acción Local que se sumaron a la iniciativa y 12 los que lo hicieron al Programa Operativo para el Desarrollo y Diversificación Económica de Zonas Rurales (PRODER) puesto en marcha por el Estado español a partir de 1996. En el Período de Programación 2000-2006 el número de GAL se amplió a 24 y ese es el número que se mantiene en la actualidad, agrupando a la totalidad de los municipios extremeños con la única excepción de las ciudades de Badajoz, Cáceres, Mérida y Plasencia (Mapa 1).

La configuración y caracterización de los GAL extremeños manifiestan un elevado grado de diversidad y muestra la existencia de marcados contrastes en lo concerniente a su extensión, volumen poblacional, densidad y número de municipios, circunstancias éstas que no siempre mantienen proporcionalidad con el montante total de fondos invertidos en los correspontientes territorios (Tabla 1). Tales diferencias obedecen a la variedad geográfica propia de la región extremeña, conformada por comarcas que se asientan sobre terrenos de montaña, otras localizadas sobre las extensas áreas de penillanura y otras, las menos, sobre los depósitos sedimentarios de vegas y depresiones.

Esta diversidad territorial dificulta la evaluación del grado de eficiencia con que cada uno de los GAL ha gestionado los fondos comunitarios asignados, entorpece determinar cuáles han conseguido mayores objetivos socioeconómicos o cuáles han logrado incrementar en mayor medida su capital social y conseguir un mayor grado de fijación de la población sobre el territorio. El volumen de información que debe manejarse para conseguir estos objetivos es ingente y la cantidad de parámetros que es conveniente utilizar es, igualmente, elevada. De ahí, precisamente, que el objetivo principal del presente artículo no se centre en realizar una evaluación de la gestión realizada por los GAL extremeños en el período de programación 2007-2013 (Márquez et al., 2005; González, J., 2006; Cejudo y Navarro, 2012), sino que lo que pretende es utilizar estos resultados para tratar de valorar el grado de idoneidad alcanzado en la implementación de las políticas de desarrollo rural y, con ello, sopesar la pertinencia o no de realizar ajustes territoriales en todos o en algunos de los GAL regionales. Lo que se intenta, en definitiva, es valorar si el tamaño y la caracterización de los grupos, tanto en términos territoriales como poblacionales, es el más adecuado o si, por el contrario, es posible realizar algunos reajustes que posibiliten optimizar la inversión de fondos públicos en el territorio. 


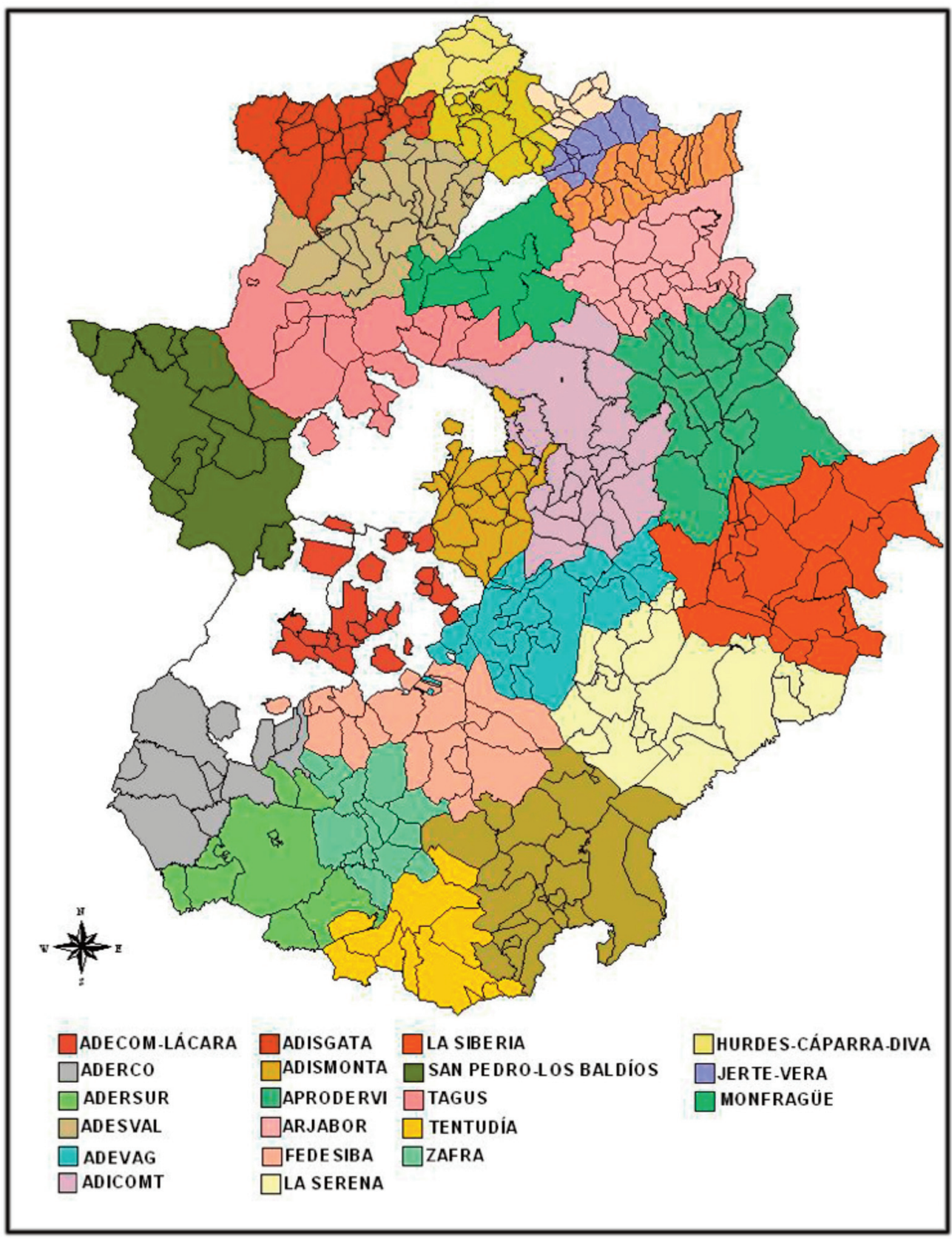

Mapa 1. Grupos de Acción Local de Extremadura. Configuración Territorial 2007-2013. Fuente: Elaboración propia. 
Tabla 1. Datos generales de los GAL (2007-2013)

\begin{tabular}{|l|c|c|c|c|c|}
\hline GAL ${ }^{2}$ & $\begin{array}{c}\text { Población } \\
(2013)\end{array}$ & Superficie & Densidad & Municipios & $\begin{array}{c}\text { Inversión } \\
\text { ejecutada }\end{array}$ \\
\hline Adecom-Lácara & 61.970 & 820,5 & 75,5 & 21 & 5.749 .969 \\
Ademe & 9.504 & $1.175,6$ & 8,1 & 7 & 5.454 .204 \\
Aderco & 32.406 & $1.647,4$ & 19,7 & 11 & 7.962 .061 \\
Adersur & 31.126 & $1.527,8$ & 20,4 & 10 & 8.032 .941 \\
Adesval & 38.362 & $1.753,6$ & 21,9 & 27 & 4.964 .497 \\
Adevag & 88.410 & $1.652,7$ & 53,5 & 17 & 7.611 .396 \\
Adic-Hurdes & 6.338 & 449,4 & 12,7 & 6 & 4.921 .502 \\
Adicomt & 32.134 & $2.355,5$ & 13,6 & 20 & 8.374 .008 \\
Adicover & 24.886 & 883,1 & 28,2 & 19 & 8.393 .091 \\
Adisgata & 21.933 & $1.257,9$ & 17,4 & 20 & 7.178 .432 \\
Adismonta & 18.481 & 962,6 & 19,2 & 22 & 5.888 .970 \\
Aprodervi & 13.719 & $2.546,5$ & 5,4 & 19 & 6.728 .993 \\
Arjabor & 38.134 & $1.491,9$ & 25,6 & 23 & 9.304 .784 \\
Campiña Sur & 31.583 & $2.699,0$ & 11,7 & 21 & 8.859 .606 \\
\hline
\end{tabular}

2. ADECOM-LÁCARA: Asociación para el desarrollo de la comarca de Lácara. ADEME: Asociación para el desarrollo de Monfragüe y su entorno. ADERCO: Asociación para el desarrollo rural de la comarca de Olivenza. ADERSUR: Asociación para el desarrollo rural Jerez-Sierra Suroeste. ADESVAL: Asociación para el desarrollo del Valle del Alagón. ADEVAG: Asociación para el desarrollo de Vegas Altas del Guadiana. ADIC-HURDES: Asociación para el desarrollo integral de la comarca de Las Hurdes. ADICOM: Asociación para el desarrollo interal de la comarca de Miajadas-Trujillo. ADICOVER: Asociación para el desarrollo integral de la comarca de La Vera. ADISGATA: Asociación para el desarrollo integral de Sierra de Gata. ADISMONTA: Asociación para el desarrollo integral de Sierra de Montánchez y Tamuja. APRODERVI: Asociación para la Promoción y el Desarrollo Rural de las Villuercas, Ibores y La Jara. ARJABOR: Asociación para el Desarrollo de la Comarca del Campo Arañuelo. CAMPIÑA SUR: Centro de Desarrollo Rural "Campiña Sur". CÁPARRA: Asociación para el Desarrollo de la Comarca de Trasierra-Tierras de Granadilla. CEDECO TENTUDÍA: Centro de Desarrollo Comarcal de Tentudía. CEDER LA SERENA: Consorcio Centro de Desarrollo Rural "La Serena". CEDER LA SIBERIA: Centro de Desarrollo Rural La Siberia. DIVA: Asociación para el Desarrollo Integral del Valle del Ambroz. FEDESIBA: Federación para el Desarrollo de Sierra Grande-Tierra de Barros. SAN PEDRO-BALDÍOS: Asociación para el Desarrollo Integral de Sierra de San Pedro-Los Baldíos. SOPRODEVAJE: Sociedad para la Promoción y Desarrollo del Valle del Jerte. TAGUS: Asociación para el Desarrollo Integral de la Comarca del Tajo-SalorAlmonte. ZAFRA-RÍO BODIÓN: Asociación Centro de Desarrollo Rural Zafra-Río Bodión. 
Tabla 1. Datos generales de los GAL (2007-2013) (continuación)

\begin{tabular}{|l|c|c|c|c|c|}
\hline GAL & $\begin{array}{c}\text { Población } \\
(2013)\end{array}$ & Superficie & Densidad & Municipios & $\begin{array}{c}\text { Inversión } \\
\text { ejecutada }\end{array}$ \\
\hline Cáparra & 9.115 & 707,8 & 12,9 & 15 & 5.930 .316 \\
Cedeco Tentudía & 20.833 & $1.284,0$ & 16,2 & 9 & 7.310 .732 \\
Ceder La Serena & 41.069 & $2.784,7$ & 14,7 & 19 & 9.490 .188 \\
Ceder La Siberia & 25.196 & $2.942,9$ & 8,6 & 18 & 7.660 .147 \\
Diva & 7.967 & 234,2 & 34,0 & 8 & 6.563 .004 \\
Fedesiba & 84.773 & $1.922,9$ & 44,1 & 19 & 12.147 .939 \\
San Pedro-Baldíos & 24.604 & $2.547,8$ & 9,7 & 12 & 6.494 .724 \\
Soprodevaje & 11.181 & 374,3 & 29,9 & 11 & 6.697 .052 \\
Tagus & 27.327 & $2.176,1$ & 12,6 & 15 & 9.518 .175 \\
Zafra-Río Bodión & 47.108 & $1.112,2$ & 42,4 & 15 & 7.221 .467 \\
\hline Total & 748.159 & $37.310,1$ & 557,7 & 384 & 178.458 .197 \\
\hline
\end{tabular}

Fuente: INE, REDEX (Elaboración propia).

\section{Metodología}

Para valorar en qué medida la configuración actual de los GAL extremeños resulta la más adecuada para gestionar los fondos comunitarios con mayores niveles de eficacia y eficiencia, se ha seguido una metodología consistente, en primer lugar, en un estudio de la situación actual de los veinticuatro Grupos de Acción Local regionales atendiendo a los resultados del Programa Operativo 2007-2013. Tras dicho estudio, se procedió a aplicar un análisis multifactorial con el objetivo de facilitar la interpretación de esa compleja realidad y, en consecuencia, valorar la conveniencia de mantener o modificar las estructuras territoriales que han servido para la gestión y aplicación de las inversiones realizadas durante el Período de Programación 2007-2013.

La utilización del Análisis Factorial ha mostrado su utilidad en los más diversos campos científicos. En el ámbito geográfico, se ha aplicado ampliamente en los estudios demográficos (Aguilera y González, 1988; Ávila, 1993; Ocaña y Navarro, 2001; Vidal et al., 2008; Fernández, 2009; Sánchez, 2009; Aguilar, 2013; Emma et al., 2014), y con especial profusión en temas territoriales y cuestiones de índole económica (Saco, 1995; Díaz, 1996; Rua et al., 2003; Serra et al., 2006; Méndez et al., 2006; Jordá et al., 2013; Arias et al., 
2016), sin que haya sido infrecuente su uso en análisis de tipo urbanístico (Altuzarra, 2010) o medioambiental (Aguilera y Garrido, 2001; García et al., 2014).

Debido a las características de la información manejada, se ha estimado oportuno el empleo del Método de Componentes Principales (PCA), con el objeto de reducir las 22 variables contempladas a unos pocos componentes principales, que constituirán una combinación lineal de las variables originales y estarán intercorrelacionadas. Se trata, en definitiva, de reducir la amplia matriz de datos iniciales a otra menor, con lo que se simplifica el estudio de una información muy voluminosa sin perder más que una parte poco significativa de la misma (Cuadras, 1981; Martens y Naes, 1989; Santos Preciado, 1991; Chuvieco, 1996).

El primer paso para ejecutar esta técnica fue la confección y posterior depuración de la Base de Datos Primaria, pues se constató que las variables disponibles para su utilización en el Análisis en Componentes Principales eran muy distintas en sus valores finales (tantos por cientos, tantos por mil, valores absolutos, etc.), por lo que se requería una tipificación de tales variables expresando las mismas en desviaciones respecto a la media, evitando así problemas derivados de la escala.

La normalización o ponderación de variables se ha realizado diviendo el valor original de cada variable y cada caso por la desviación típica de esa variable para el conjunto de casos. De esa manera todas las variables están en el mismo orden de magnitud, con independencia de las unidades de medida originalmente utilizadas, de manera que a la hora de realizar el Análisis en Componentes Principales (PCA) todas las variables tienen un "equilibrio de fuerzas" matemático, y las de mayor valor absoluto en unidades de medida no pesan más que las que tienen menos valor numérico.

Por otro lado, en el proceso factorial llevado a cabo mediante el método de Componentes Princiales, se han seleccionado aquellos componentes que recogen el porcentaje de variabilidad suficiente (varianza igual o superior a 1) como para explicar el fenómeno en cuestión. Ello se entiende así puesto que la varianza de cada variable (que es tanto como decir la diversidad, en esas variables, de los diversos lugares) es igual a la unidad, debido a que en la matriz de puntuaciones la variables se presentan tipificadas (media nula y varianza unitaria) (Santos Preciado, 1991).

La ecuación esencial del Análisis en Componentes Principales es de tipo lineal en donde los componentes se agregan aditivamente:

$$
\mathrm{Z}_{\mathrm{ji}}=\mathrm{A}_{\mathrm{j} 1} \mathrm{~F}_{1 \mathrm{i}}+\mathrm{A}_{\mathrm{j} 2} \mathrm{~F}_{2 \mathrm{i}}+\ldots+\mathrm{A}_{\mathrm{jm}} \mathrm{F}_{\mathrm{mi}}
$$

Previamente, dado que se manejaban un buen número de variables referentes a los 24 GAL, se procedió a una reducción progresiva de la matriz de puntuaciones mediante análisis estadísticos exploratorios. Éstos consistieron en la realización de varios análisis factoriales con un número de variables mucho mayor; detectándose en ellos que muchas de las variables eran redundantes, esto es, tendían a explicar el mismo fenómeno. 
Una vez realizado el análisis factorial definitivo fueron seleccionados los componentes principales y se procedió a representarlos en forma de matriz. En este sentido, cada elemento de esta matriz representa los coeficientes factoriales de las variables, es decir, las correlaciones entre las variables y los componentes principales. La matriz, por tanto, tendrá tantas columnas como componentes principales y tantas filas como variables.

\section{Valoración de los Grupos de Acción Local (2007-2013).}

Durante el período de programación 2007 y hasta fecha de 30 de septiembre de 2014, según los datos proporcionados por la Dirección General de Desarrollo Rural del Gobierno de Extremadura, las inversiones realizadas alcanzaron un total de $92.788 .575,92 €$ de subvención pública y 78.470.900,98 € de inversiones privadas, alcanzando un monto de 171.259.476,90 €. Esa relación entre la subvención pública y la inversión privada tiene un comportamiento distinto en cada Grupo de Acción Local en función de las medidas prioritarias que cada grupo haya estimado conveniente para el período de programación. En estos datos no se aprecia una distribución lógica de la subvención pública entre los distintos Grupos de Acción Local, esto es, no existe relación alguna entre, por ejemplo, la población total de los GAL y la subvención recibida, es más la correlación existente (Coeficiente de Pearson) entre la variable "Población de 2013" y la variable "Subvención pública/habitante (en euros)" es negativa ( $r=-0.760$ ), ello significa que a medida que aumenta la variable población disminuye la variable subvención.

Al mismo tiempo, también existe una correlación negativa entre la variable "Tamaño superficial del GAL ( $\mathrm{km}^{2}$ )" y la variable "Subvención pública/habitante (en euros)", suponiendo ésta una correlación negativa de $r=-0.548$. Ambas realidades confirman que ha habido una mayor subvención pública en aquellos Grupos de Acción Local con menor tamaño poblacional y con menor superficie territorial.

En segundo lugar, los Grupos de Acción Local con mayor tamaño poblacional consiguen tener unos porcentajes más altos de inversiones privadas que los de menor tamaño poblacional. En este caso, la correlación entre la variable "Población de 2013" y la "Inversión privada total" es positiva, alcanzo una correlación de $r=0.584$. Ello, sin duda, no hace sino poner de relieve la importancia que tiene el tamaño poblacional y el dinamismo económico. En este sentido, los GAL con porcentajes más altos de inversiones privadas frente a la subvención pública recibida son Adecom-Lácara (58,67\%), Adicomt (51,03\%), Fedesiba (59,39\%) y Tagus (50,24\%), estando próximos al 50\% Aderco (49,37\%) y Adersur (49,49\%). El resto sucesivamente se aleja del 50/50, primando en todo caso la subvención pública frente a la inversión privada. Cabe reseñar que en los GAL de Zafra-Río Bodión y Adevag, pese a tener un importante dinamismo demográfico y económico, la subvención pública ha estado muy por encima de la inversión privada en el último programa operativo, 33,56\% y 37,99\% respectivamente. 
Una partida económica importante en la gestión de los Grupos de Acción Local está destinada a los equipos técnicos que velan por el correcto cumplimiento y funcionamiento del propio grupo. La distribución de los gastos corrientes destinados a la Medida 431 "Funcionamiento de los Grupos de Acción Local”, permite apreciar unos valores porcentuales medios del 17\%, si bien hay una fuerte desviación entre aquellos que destinan menos fondos para estos gastos (caso de Adicover, que sólo emplaza un 6,9\%) o aquellos otros que destinan más de un 20\% a dichos gastos (caso de Aprodervi con un 22,9\%, Tentudia con un 20,7\%, Aderco con un 20,8\%, Adic-Hurdes con un 20,4\% y Adesval con un 20,1\%).

Cuando se analizan y relacionan los gastos de funcionamiento en cada Grupo de Acción Local con los habitantes de cada uno de los territorios se observa también que hay una clara correlación negativa entre el número de habitantes y los gastos de funcionamiento, esto es, cuanto menor es el tamaño poblacional mayores son los gastos de funcionamiento, aspecto en todo caso contradictorio. El coeficiente de correlación es, en este caso, de $r=0.717$.

Otro aspecto a considerar es la distribución de los gastos por medidas en el conjunto del territorio. Como se puede comprobar en la Tabla 2, la Medida 413 sobre "Estrategias de desarrollo local: Calidad de vida y diversificación de la economía" es la que acapara en general un porcentaje más alto de subvención pública total (72,5\%), seguida de la Medida 431 sobre "Funcionamiento de los Grupos de Acción Local" que acapara un 17\%. Curiosamente, en la Medida 411 sobre "Estrategias desarrollo local sobre competitividad" sólo acumula un 10\% de la subvención pública total. En todo caso, es necesario señalar que la Medida 411 es la que mayor porcentaje de inversión privada supone frente a la subvención pública, cifrándose en 1,7 euros de inversión privada por 1 euro de subvención pública.

Tabla 2. Distribución de los gastos por medidas (en euros)

\begin{tabular}{|l|c|c|c|c|r|}
\hline Medidas & Subvención Pública & $\%$ & Inversión Privada & $\%$ & \multicolumn{1}{|c|}{ Total } \\
\hline 411 & $9.246 .427,1$ & 37,1 & $15.703 .915,1$ & 62,9 & $24.950 .342,2$ \\
413 & $67.254 .272,2$ & 51,9 & $62.357 .646,1$ & 48,1 & $129.611 .918,2$ \\
421 & $608.883,3$ & 88,0 & $82.860,7$ & 12,0 & $691.743,9$ \\
431 & $15.678 .993,5$ & 98,0 & $326.479,2$ & 2,0 & $16.005 .472,7$ \\
\hline Total medidas & $92.788 .576,0$ & 54,2 & $78.470 .901,0$ & 45,8 & $171.259 .477,0$ \\
\hline
\end{tabular}

Fuente: Dirección General de Desarrollo Rural (GOBEX).

411. Estrategias de desarrollo local sobre competitividad

413. Estrategias de desarrollo local: Calidad de vida y diversificación de la economía

421. Cooperación trasnacional e interregional

431. Funcionamiento de los Grupos de Acción Local 
Cabría incidir que la Medida 421 sobre "Cooperación transnacional e interregional" ha sido ciertamente irrelevante por cuanto que sólo ha supuesto un $0,6 \%$ del total de la subvención pública.

Por otra parte, la distribución de los gastos por medidas y submedidas es totalmente distinta en cada Grupo de Acción Local, pese a que algunos podían tener las mismas estrategias de desarrollo por cuanto que los recursos y las potencialidades son similares.

De este modo, la Medida 413 sobre "Estrategias de desarrollo local: Calidad de vida y diversificación de la economía" es la que mayor porcentaje de gasto acumula en todos y cada uno de los Grupos de Acción Local. Sin embargo, seguidamente ya no se encuentra exclusivamente a la Medida 431 sobre "Funcionamiento de los Grupos de Acción Local", sino que también en algunos casos aparece la Medida 411 sobre "Estrategias desarrollo local sobre competitividad" como la segunda más importante en los gastos de subvención pública. En este caso figuran los Grupos de Acción Local de Ademe, Adersur, Adicover, La Serena y Soprodevaje. Grupos estos que, de una u otra manera, han destinado un alto porcentaje de la subvención pública a la submedida 123 sobre "Aumento del valor añadido de los productos agrícolas y forestales", relacionados en todo caso con el sector agroindustrial.

Por lo tanto, se puede afimar en primer lugar que absolutamente todos los Grupos de Acción Local han destinado la mayor parte de la subvención pública a submedidas incluidas dentro de la Medida 413. En segundo lugar que, a excepción de los GAL anteriormente citados, una gran mayoría destinan en un alto porcentaje a los gastos de funcionamiento (Medida 431) y que, en tercer lugar, la Medida 421 es francamente testimonial y que, por tanto, los gastos destinados a la cooperación trasnacional e interregional han sido mínimos y, en todo caso, puntuales en algunos GAL como Aderco, Adic-Hurdes, Adicomt, Aprodervi, San Pedro-Los Baldios y Tentudia que superan escasamente el 1\% de la subvención pública.

Por último, los Grupos de Acción Local tienen un comportamiento muy diferente en lo referente a la captación de inversiones privadas frente a las subvenciones públicas recibidas, independientemente de las medidas o submedidas de las que se trate. En todo caso, este dato muestra aquellos GAL en los que se consiguen mayores inversiones privadas por cada 100 euros de subvención pública recibida. Los grupos de Fedesiba y Adecom-Lácara son los que consiguen una mayor ratio de inversión privada sobre la subvención recibida en total, 146 euros en el caso del primero y 142 el segundo. Próximos a una ratio de 100, pero por encima de la misma, se encuentran Adicomt y Tagus. Próximos a 100 pero por debajo de ese umbral estarían Adersur y Aderco. Los GAL con menor tamaño poblacional consiguen menos euros de inversión privada sobre la subvención recibida, así estos últimos lugares lo ocupan Ademe (62 euros), Soprodevaje (57 euros), Adic-Hurdes (49 euros) o Adismonta (49 euros). Si es sorprendente, por otro lado, que GAL con suficiente masa poblacional crítica como Adevag (61 euros) o Zafra-Río Bodión (50 euros) ocupen los últimos lugares. 
Igualmente este comportamiento también se observa cuando se analiza esa ratio por Medidas. De esta manera, la Medida 441 sobre "Estrategias de desarrollo local sobre competitividad", netamente de carácter productivo, presenta unos valores muy distintos en cada Grupo de Acción Local. Es así como los grupos de Adecom-Lácara (205 euros), Adicomt (209 euros), Fedesiba (218 euros), La Siberia (260 euros) o Tagus (213 euros) han conseguido unas inversiones privadas muy altas sobre una base de 100 euros de subvención pública. En cambio, los GAL de Adic-Hurdes (122 euros), Adismonta (128 euros) o Cáparra (114 euros) son algunos de los grupos que menor ratio alcanzan de inversión privada frente a la subvención pública recibida en esta Medida.

En cambio, la Medida 413 sobre "Estrategias de desarrollo local: Calidad de vida y diversificación de la economía", al contar con submedidas de distinto carácter (competitivas y no competitivas, algunas de éstas últimas subvencionables hasta un cien por cien) reflejan que la mayor parte de los Grupos de Acción Local arrojan ratios inferiores a 100, esto es, frente a 100 euros de subvención pública no se alcanza esa cifra de inversión privada. De esta manera, grupos como Adecom-Lácara, Fedesiba, Aderco, Tagus, Adicomt, Tentudía, incluso Aprodervi y Campiña Sur, están por encima de 100 euros de inversión privada frente a 100 euros de subvención pública recibida.

En cambio, hay una serie de grupos que, en todo caso, han decidido primar en su estrategia de desarrollo aquellas submedidas relacionadas con los "Servicios básicos para la economía y la población rural" (Submedida 321), con la "Renovación y desarrollo de poblaciones rurales" (Submedida 322), con la "Conservación y mejora del patrimonio rural" (Submedida 323), con la "Formación e información de agentes económicos" (Submedida 331) o con la Adquisición de capacidades y promoción" (Submedida 341). Dentro de estos grupos cabría citarse a Ademe (35 euros), Adic-Hurdes (54 euros), Adismonta ( 49 euros), Soprodevaje (44 euros) o Zafra-Río Bodión (55 euros); en todos ellos, la inversión privada está muy por debajo de la subvención pública destinada a estas submedidas.

En virtud de estas consideraciones de partida y, con el objetivo de relacionar esta diversidad de resultados con la conformación y caracterización de los GAL, quedó confeccionada la base de datos, como se refirió en el apartado metodológico, con aquellos indicadores que se han considerado fundamentales para valorar la configuración y los resultados alcanzados por los GAL tras la finalización del período de programación 2007-13. De este modo, la matriz de puntuaciones (lugares por variables) quedó confeccionada en los siguientes términos dentro de la siguiente tabla 3.

Tras realizar un primer Análisis en Componentes Principales sobre la matriz de puntuaciones reseñada (Tabla 3), se obtuvieron unos resultados muy satisfactorios, puesto que entre los tres primeros componentes se alcanzaba a explicar más del $80 \%$ de la varianza total. En particular los dos primeros componentes ya superan el $75 \%$ de la varianza explicada, por lo que la interpretación de la relación entre variables se ha realizado a partir de ellos. 
Tabla 3. Matriz de Puntuaciones Primaria

\begin{tabular}{|c|c|c|}
\hline Variables & Descripción & Extracción \\
\hline $\begin{array}{l}\mathrm{X}_{1} \text { : Población joven } \\
\text { (menores de } 15 \text { años) }\end{array}$ & $\begin{array}{l}\text { № de personas menores de } 15 \text { años } \\
\text { respecto de la población total (2013) }\end{array}$ & 0,972 \\
\hline $\begin{array}{l}\mathrm{X}_{2} \text { : Población adulta } \\
\text { (entre } 15 \text { y } 64 \text { años) }\end{array}$ & $\begin{array}{l}\text { № de personas entre } 15 \text { y } 64 \text { años } \\
\text { respecto de la población total (2013) }\end{array}$ & 0,954 \\
\hline $\begin{array}{l}\mathrm{X}_{3}: \text { Población anciana } \\
\text { (mayor de } 65 \text { años) }\end{array}$ & $\begin{array}{l}\text { № de personas mayores de } 65 \text { años } \\
\text { respecto de la población total (2013) }\end{array}$ & 0,984 \\
\hline $\begin{array}{l}\mathrm{X}_{4} \text { : Densidad de población } \\
\text { (habitantes } / \mathrm{km}^{2} \text { ) }\end{array}$ & № de habitantes por $\mathrm{km}^{2}$ (2013) & 0,702 \\
\hline $\begin{array}{l}\mathrm{X}_{5}: \text { Tasa de Crecimiento } \\
\text { Real (2001-2013) }\end{array}$ & $\begin{array}{l}\text { Porcentaje de población } 2013 \\
\text { sobre la de } 2001\end{array}$ & 0,866 \\
\hline $\begin{array}{l}X_{6} \text { : Razón de Feminidad } \\
(49 \text { años) }\end{array}$ & $\begin{array}{l}\text { Porcentaje de mujeres con edades } \\
\text { entre } 15-49 \text { años por cada } 100 \text { hombres } \\
\text { con tal edad (2013) }\end{array}$ & 0,809 \\
\hline $\mathrm{X}_{7}$ : Índice de Infancia & $\begin{array}{l}\text { Porcentaje de menores de } 15 \text { años } \\
\text { sobre la población total (2013) }\end{array}$ & 0,765 \\
\hline $\mathrm{X}_{8}$ : Tasa de Maternidad & $\begin{array}{l}\text { Niños menores de } 5 \text { años por cada } 100 \\
\text { mujeres entre } 15 \text { y } 49 \text { años (2013) }\end{array}$ & 0,900 \\
\hline $\mathrm{X}_{9}$ : Tasa Bruta de Natalidad & $\begin{array}{l}\text { Nacidos por cada } 1.000 \text { habitantes } \\
(2008-2012)\end{array}$ & 0,933 \\
\hline $\mathrm{X}_{10}$ : Tasa Bruta de Mortalidad & $\begin{array}{l}\text { Defunciones por cada } 1.000 \text { habitantes } \\
\text { (2008-2012) }\end{array}$ & 0,914 \\
\hline $\mathrm{X}_{11}:$ Índice de Envejecimiento & $\begin{array}{l}\text { Porporción de mayores de } 65 \text { años por } \\
\text { cada } 100 \text { menores de } 15 \text { (2013) }\end{array}$ & 0,939 \\
\hline $\begin{array}{l}\mathrm{X}_{12}: \text { № de medio de } \\
\text { habitantes por núcleo }\end{array}$ & $\begin{array}{l}\text { Promedio de habitantes por núcleo } \\
\text { de población (2013) }\end{array}$ & 0,867 \\
\hline $\begin{array}{l}\mathrm{X}_{13} \text { : Tasa de Dependencia } \\
\text { de Mayores }\end{array}$ & $\begin{array}{l}\text { Proporción de mayores sobre la Población } \\
\text { Potencialmente Activa (15-64 años) (2013) }\end{array}$ & 0,901 \\
\hline $\begin{array}{l}\mathrm{X}_{14}: \text { Tasa de Reemplazo de } \\
\text { Población Potencialmente Activa }\end{array}$ & $\begin{array}{l}\text { Porcentaje de población de } 24 \text { años sobre } \\
\text { la población de 55-64 años (2013) }\end{array}$ & 0,901 \\
\hline $\begin{array}{l}X_{15}: \text { Afiliados a la Seguridad } \\
\text { Social, } 2013 \text { (Sector Agrario) }\end{array}$ & $\begin{array}{l}\text { Porcentaje de afiliados a la Seguridad } \\
\text { Social en el Sector Agrario (2013) }\end{array}$ & 0,989 \\
\hline $\mathrm{X}_{16}:$ Afiliados (Sector Industrial) & $\begin{array}{l}\text { Porcentaje de afiliados a la Seguridad } \\
\text { Social en el Sector Industrial (2013) }\end{array}$ & 0,784 \\
\hline
\end{tabular}




\begin{tabular}{|l|l|c|}
\hline Variables & Descripción & Extracción \\
\hline $\begin{array}{l}\mathrm{X}_{17}: \text { Afiliados } \\
\text { (Sector Construcción) }\end{array}$ & $\begin{array}{l}\text { Porcentaje de afiliados a la Seguridad } \\
\text { Social en el Sector Construcción (2013) }\end{array}$ & 0,757 \\
$\mathrm{X}_{18}:$ Afiliados (Sector Servicios) & $\begin{array}{l}\text { Porcentaje de personas afiliadas en la } \\
\text { Seguridad Social al Sector Servicios (2013) }\end{array}$ & 0,960 \\
$\mathrm{X}_{19}:$ Índice de Actividades & $\begin{array}{l}\text { Índice comparativo del conjunto de la } \\
\text { actividad económica, se obtiene en } \\
\text { Económicas } \\
\text { función del impuesto correspondiente } \\
\text { al total de actividades económicas } \\
\text { empresariales (2009) }\end{array}$ & 0,581 \\
$\mathrm{X}_{20}:$ Tierras Labradas & $\begin{array}{l}\text { Porcentaje de tierras labradas (2009) } \\
\text { Porcentaje de tierras no labradas (2009) }\end{array}$ & 0,963 \\
$\mathrm{X}_{21}:$ Tierras No Labradas & $\begin{array}{l}\text { Población absoluta a 1 de Enero } \\
\text { de 2013 (INE) }\end{array}$ & 0,904 \\
$\mathrm{X}_{22}:$ Población absoluta (2013) & \\
\hline
\end{tabular}

Fuente: INE y elaboración propia.

Tabla 4. Varianza explicada y acumulada por componentes

\begin{tabular}{|c|c|c|}
\hline Componentes Principales & \% Varianza Explicada & \% Varianza Acumulada \\
\hline 1 & 62,52 & 62,52 \\
2 & 12,74 & 75,26 \\
3 & 7,51 & 82,77 \\
4 & 5,95 & 88,71 \\
5 & 3,24 & 91,95 \\
6 & 2,23 & 94,18 \\
7 & 1,99 & 96,17 \\
\hline
\end{tabular}

Fuente: INE y elaboración propia.

El gráfico de cargas factoriales (Gráfico 1), permite visualizar fácilmente la relación existente entre las variables utilizadas. En este sentido, aquellas que están próximas mantienen entre sí elevadas correlaciones positivas, en tanto que las más alejadas tienen correlaciones negativas. 
Tabla 5. Cargas factoriales por variables y componentes principales

\begin{tabular}{|l|c|c|c|}
\hline Variables & $P C-1$ & $P C-2$ & $P C-3$ \\
\hline Población joven (menores de 15 años) & 0,26136 & $-0,02957$ & 0,10725 \\
Población adulta (entre 15 y 64 años) & 0,25473 & 0,03106 & 0,17627 \\
Población anciana (mayor de 65 años) & $-0,26256$ & $-0,00306$ & $-0,14624$ \\
Densidad de población (habitantes/km2) & 0,19011 & 0,18620 & $-0,04445$ \\
Tasa de Crecimiento Real (2001-2013) & 0,21237 & 0,05107 & 0,25352 \\
Relación de Feminidad (49 años) & 0,21790 & $-0,21145$ & $-0,13027$ \\
Índice de Infancia & 0,26135 & $-0,02735$ & 0,10904 \\
Tasa de Maternidad & 0,25502 & $-0,03446$ & 0,02370 \\
Tasa Bruta de Natalidad & 0,25919 & 0,02164 & 0,06638 \\
Tasa Bruta de Mortalidad & $-0,23221$ & $-0,12638$ & $-0,34797$ \\
Índice de Envejecimiento & $-0,25404$ & 0,05754 & $-0,13196$ \\
№ de medio de habitantes por municipio & 0,24455 & 0,02576 & $-0,15791$ \\
Tasa de Dependencia de Mayores & 0,22172 & $-0,09710$ & $-0,04011$ \\
TRPPA & 0,22172 & $-0,09710$ & $-0,04011$ \\
Afiliados a la S.S., 2013 (Sector Agrario) & $-0,06026$ & 0,55549 & 0,12483 \\
Afiliados a la S.S., 2013 (Sector Industrial) & 0,06837 & $-0,46577$ & 0,22656 \\
Afiliados a la S.S., 2013 (Sector Construcción) & $-0,17043$ & $-0,24068$ & 0,08359 \\
Afiliados a la S.S., 2013 (Sector Servicios) & 0,09346 & $-0,45868$ & $-0,32133$ \\
Índice de Actividades Económicas & 0,14972 & 0,02086 & 0,22151 \\
Tierras Labradas & 0,19822 & 0,17989 & $-0,44286$ \\
Tierras No Labradas & $-0,19822$ & $-0,17989$ & 0,44286 \\
Población absoluta (2013) & 0,23204 & 0,08658 & $-0,22551$ \\
\hline
\end{tabular}

Fuente: INE y elaboración propia.

El primer componente retiene casi el 63\% de la varianza total. Con alta carga factorial positiva, y situadas muy próximas, lo que indica su elevada correlación y que vienen a medir lo mismo, tenemos las variables: 
$X_{1}$ : Población joven (menores de 15 años)

$X_{2}$ Población adulta (entre 15 y 64 años)

$X_{5}$ : Tasa de Crecimiento Real (2001-2013)

$X_{6}:$ Relación de Feminidad (49 años)

$X_{7}$ Índice de Infancia

$X_{8}$ :Tasa de Maternidad

$X_{9}$ : Tasa Bruta de Natalidad

$X_{12}$ № de medio de habitantes por municipio

$X_{13}$ : Tasa de Dependencia de Mayores

$X_{14}$ Tasa de Reemplazo de Población Potencialmente Activa

$X_{22}$ Población absoluta (2013)

Próximas a este grupo, pero con menor carga, podemos observar en el plano positivo las variables:

$X_{4}$ : Densidad de población (habitantes $/ \mathrm{km}^{2}$ )

$X_{19}$ : Índice de Actividades Económicas (2009)

$X_{20}$ :Tierras Labradas

Por otra parte, con alta carga factorial negativa se encuentran las variables:

$X_{3}$ : Población anciana (mayor de 65 años)

$X_{10}$ :Tasa Bruta de Mortalidad

$X_{11}:$ Indice de Envejecimiento

$X_{17}$ : Afiliados a la Seguridad Social, 2013 (Sector Construcción)

$X_{21}$ : Tierras No Labradas

Por lo tanto, este primer componente separa y define perfectamente las variables relacionadas básicamente con la demografia.

El segundo componente retiene el 13\% de la variación inicial. Separa con elevada carga positiva (parte superior del Gráfico 1), la variable $X_{15}$ : Afiliados a la Seguridad Social (Sector Agrario), en contraposición a las variables $X_{18}$ : Afiliados a la Seguridad Social (Sector Servicios) y $X_{16}$ : Afiliados a la Seguridad Social (Sector Industria), ambas con elevada carga negativa (parte inferior del gráfico), a su vez muy cercanas y correlacionadas entre sí. Las tres variables son indicadoras del ámbito socioeconómico. 


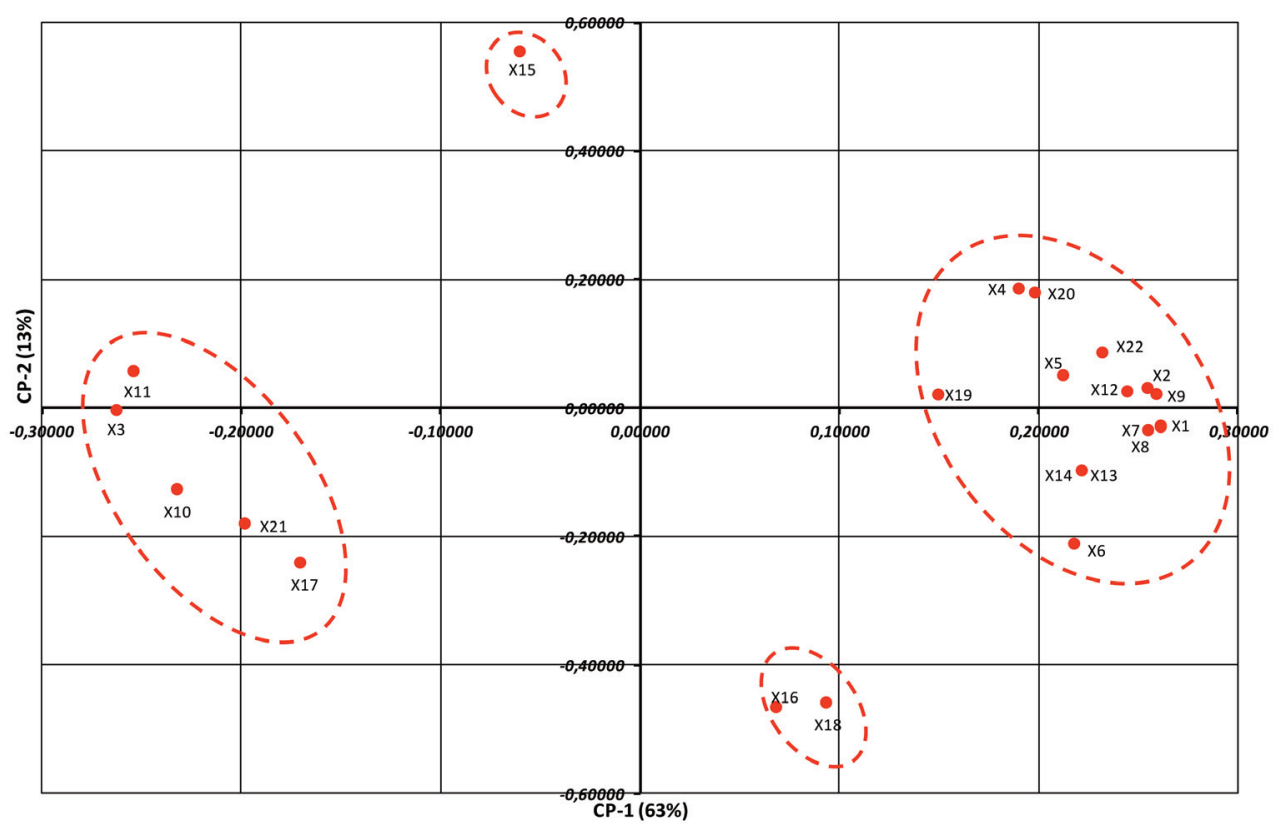

Gráfico 1. Cargas factoriales por variables.

Fuente: Elaboración propia.

La situación de los Grupos de Acción Local en el espacio relativo definido por los dos primeros componentes podemos observarla en el Gráfico 2 de Puntuaciones Factoriales.

Los Grupos de Acción Local situados en los extremos de ambos componentes son los que presentan un conjunto de características muy representativas de las variables indicadoras del Gráfico 1, en tanto que las que están más hacia el centro, tienen unas características mixtas y/o menos acusadas.

Por lo tanto, con alta puntuación positiva en el primer componente ("Grupos demográficamente progresivos"), estarían los Grupos de Acción Local de Adecom-Lácara, Fedesiba y Adevag. No muy lejos estarían los GAL de Zafra-Rio Bodión, Arjabor y Aderco.

Con puntuación negativa en el primer componente ("Grupos demográficamente regresivos”), estarían las comarcas de Adic-Hurdes, Cáparra, Diva y Aprodervi.

En cuanto al segundo componente, con carga positiva y elevado peso de la agricultura, está el Grupo de Acción Local de Soprodevaje, claramente separado del resto. Con carga negativa e importancia relativa del sector industrial y de servicios, estarían los Grupos de Acción Local de Ademe y Tagus, entre otros. 


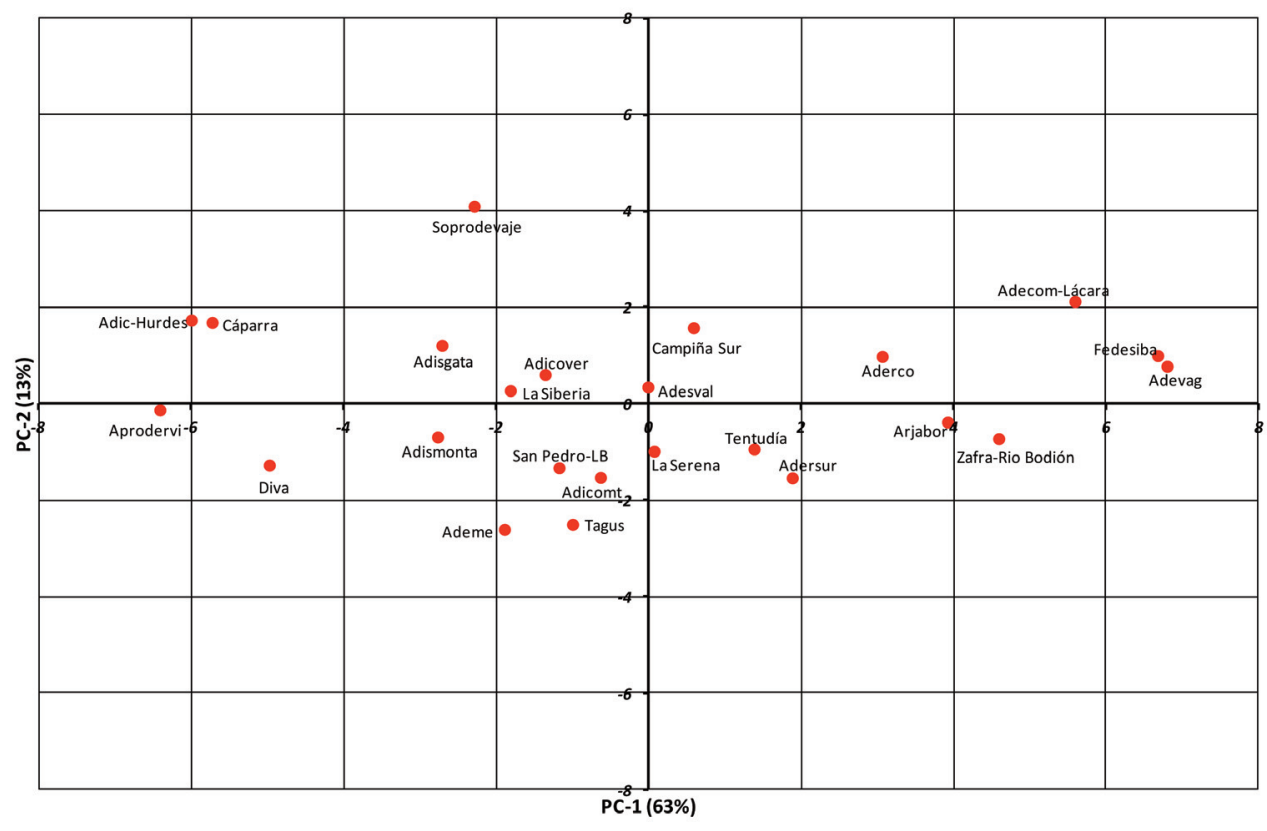

Gráfico 2. Puntuaciones factoriales de los casos.

Fuente: Elaboración propia.

El resto de Grupos de Acción Local tiene características mixtas y particulares de ambos componentes, si bien casi todos ellos tienen tendencias a la regresión demográfica y la excesiva dependencia agraria.

Por tanto, la situación socioeconómica y demográfica actual de los Grupos de Acción Local del territorio extremeño, a tenor del análisis en componentes principales realizado, tiene mucho que ver con el tamaño poblacional. Esta variable es redundante del dinamismo demográfico, esto es, cuanto mayor es la masa poblacional crítica las expectativas demográficas y, por tanto, económicas (o viceversa) de cualquier territorio mejoran sustancialmente. Por ello, los Grupos de Acción Local más regresivos demográficamente y más débiles desde el punto de vista económico son aquellos que cuentan con menor tamaño poblacional, esto es: Adic-Hurdes (6.190 habitantes), Aprodervi (13.937 habitantes), Cáparra (9.267 habitantes), Diva (7.979 habitantes), Soprodevaje (11.267 habitantes) o Ademe (9.622 habitantes), como casos más extremos.

En cambio, aquellos más dinámicos demográfica y económicamente vienen a coincidir con los GAL más poblados: Adevag (89.087 habitantes), Fedesiba (86.121 habitantes), Adecom-Lácara (62.616 habitantes), Zafra-Río Bodión (47.423 habitantes) o Arjabor (38.366 habitantes), entre otros. 


\section{Propuesta de reajustes territoriales en los GAL}

Tras la realización del presente estudio, tanto en el ámbito territorial como en el analítico estadístico, se plantea la posibilidad de realizar una serie de propuestas de configuración del territorio GAL. No es, ciertamente, una tarea fácil, puesto que se conjugan circunstancias de índole cuantitativa, cualitativa, territorial, económica, social, demográfica, política,..., que, en cada caso, pueden contribuir a justificar si no cualquier posible configuración, sí al menos varias de ellas.

\subsection{Propuesta 1 (Reagrupación por incremento poblacional)}

Partiendo del panorama actual que ofrecen los 24 GAL existentes en Extremadura y, de manera especial, teniendo en cuenta la capacidad explicativa de las variables utilizadas para su valoración y diagnóstico, bien podría inferirse que una posible opción para incrementar los niveles de eficacia y eficiencia en la implementación de la metodología LEADER, consistiría en tender progresivamente hacia la confección de Grupos de Acción Local con mayor masa poblacional crítica. Al mismo tiempo, también es obvio que es necesario buscar una menor dependencia del sector agrario y procurar una mayor diversificación económica hacia el sector industrial y de servicios. Estas premisas son fundamentales para tejer una nueva estructura territorial de los Grupos de Acción Local en Extremadura. Teniendo en cuenta las mismas, así como también otros aspectos inherentes a la continuidad geográfica, se ha intentado argumentar una primera opción consistente en la agrupación de algunos Grupos de Acción Local existentes.

Para valorar esta opción, se procedió a realizar un segundo Análisis en Componentes Principales que contemplaba "casos territoriales teóricos" con el objetivo de observar cómo sería el resultado de su implantación en un plano apriorístico.

No en vano, esta modelización ha permitido evaluar de antemano cuál sería el comportamiento de esos nuevos casos en un plano relativo de configuración territorial.

De esta manera, como en el PCA anterior, se procedió a utilizar la misma matriz de puntuaciones o base de datos, sólo que en este caso el número de lugares o Grupos de Acción Local cambiaron en parte. Las hipótesis y criterios de partida en la elección de estos cambios, pueden resumirse en los siguientes términos:

En principio, dada su situación muy satisfactoria o satisfactoria en el plano relativo del Gráfico 2, se decidió no actuar sobre los Grupos de Acción Local Adecom-Lácara, Adesval, Adevag, Adicomt, Adisgata, Adismonta, Arjabor, Campiña Sur, Fedesiba, La Serena, La Siberia, San Pedro-Los Baldíos y Tagus.

Cabe mencionar, en este sentido, que Aprodervi, pese a estar en una situación demográfica regresiva y tener poca masa poblacional crítica, tiene un elemento de co- 
hesión y participación territorial muy fuerte, en parte debido a la figura del Geoparque de Villuercas-Ibores-Jara, esto hizo que se decidiera actuar en consecuencia y optar por el mantenimiento de un GAL con unas peculiariades territoriales muy marcadas.

Por otro lado, cabe incidir que los GAL de Adisgata (22.372 habitantes) y Adismonta (18.984 habitantes) tienen una fuerte tendencia a la regresión demográfica, sin embargo la presencia de un núcleo dinámico como Moraleja, en el primer caso, o la presencia cercana de la ciudad de Cáceres, en el segundo caso, pueden suponer una importante ayuda para su desarrollo territorial.

Insistiendo, por un lado, en la certeza de que el aumento del tamaño poblacional es necesario para cambiar, en principio, la situación actual de algunos de los Grupos de Acción Local se realizan las siguientes propuestas de "nuevos casos o escenarios territoriales".

\subsubsection{Reagrupación Cáparra-Diva-Hurdes}

Esta posible agrupación se cimenta en la conveniencia de incrementar la masa crítica hasta niveles que faciliten la consecución de los objetivos de desarrollo que se planteen en el futuro. Debe tenerse en cuenta, a este respecto, que en su actual configuración, Adic-Hurdes sólo disponía en 2013 de una población de 6.190 habitantes, volumen este que quedaría reducido a 3.708 habitantes si sólo se contemplara la población potencialmente activa. En el caso de Cáparra, la población se situó en 2013 en 9.267, inferior todavía a la cifra recomendada para la delimitación de las zonas rurales. También en este caso, si sólo se tiene en cuenta la población potencialmente activa, por entender que es la que puede desempeñar el papel de actor para el desarrollo local, el volumen de población quedaría reducido a 5.560 habitantes. Finalmente, el caso de Diva responde a parámetros similares, de modo que reúne una población de 7.979 habitantes de la que sólo 4.975 tienen edades comprendidas entre 15 y 64 años.

La unión de estos tres GAL supondría incrementar su masa crítica hasta 23.436 habitantes (14.243 constituirían población potencialmente activa), lo que con toda probabilidad facilitaría alcanzar los objetivos de participación social e inversión pública y privada, en relación con la situación actual. Por otro lado, existen suficientes elementos de homogeneidad territorial como para que esta agrupación propuesta no interfiriera ni menos aún menoscabara las posibilidades de diseño de estrategias de desarrollo ni tampoco en su proximidad a los habitantes del territorio, ya que lo que se plantea es una reconfiguración territorial, lo que no debe implicar necesariamente una modificación en la estructura organizativa actual. Las propias características del territorio, con especiales dificultades de comunicación y accesibilidad, podrían aconsejar el mantenimiento de la estructura organizativa actual. 


\subsubsection{Reagrupación Soprodevaje-Adicover}

Los planteamientos para proponer esta agrupación sólo difieren parcialmente de los anteriores, ya que en este caso se trataría de reforzar el papel de Soprodevaje para lograr aproximarlo hacia niveles más positivos. Este GAL contaba en 2013 con un total de 11.267 habitantes y una "Población Potencialmente Activa" de 7.178 personas. A esta debilidad de su masa crítica deben unirse unos niveles de deterioro demográfico que se reflejan en la existencia de un "Índice de Envejecimiento" del 230,6\% (2,3 mayores de 65 años por cada niño menor de 15 años), un "Crecimiento Natural" fuertemente negativo (-4,1\%o en el período 2008-2012), un "Crecimiento Real Negativo entre 2001 y 2013 " (-4,4\% de la población) y un elevado grado de masculinización en edades fecundas (87,5 mujeres por cada 100 hombres en edades comprendidas entre los 15 y 49 años).

Adicover, por otro lado, dispone de una población de 25.248 habitantes (16.077 en el caso de la "Población Potencialmente Activa"), pero tampoco se sustrae a los signos de deterioro demográfico antes mencionados: "Índice de Envejecimiento" del 212,2\% (2,1 mayores de 65 años por cada niño menor de 15 años), un "Crecimiento Natural" fuertemente negativo (-4,3\%o en el período 2008-2012), un "Crecimiento Real Negativo entre 2001 y 2013" (pérdida del 5,2\% de la población) y masculinización en edades fecundas (91,4 mujeres por cada 100 hombres en edades comprendidas entre los 15 y 49 años).

Se estima, en consecuencia, que la unión de los dos grupos actuales (Soprodevaje y Adicover) mejoraría su posición relativa en el conjunto y, tampoco en este caso, interferiría en la elección y diseño de sus estrategias ni en su fucionamiento, dado que, como en el caso anterior, las características montañosas de los territorios aconsejarían que, para una mejor atención de los ciudadanos, se mantuviera la estructura organizativa actual, manteniendo el número de sedes actuales.

\subsection{Reagrupación por homogeneidad territorial}

No se ha querido dejar de valorar la posibilidad de procurar un mejor posicionamiento de algunos GAL en el contexto general, atendiendo a agrupaciones que tengan por objeto conseguir la reorganización de Grupos que mantengan entre sí una sustantiva homogeneidad territorial, lo que probablemente les llevaría a conseguir una mayor solidez en el diseño de sus estrategias territoriales. En este caso, se ha pretendido valorar las consecuencias que tendrían, sobre el marco general obtenido con la aplicación del primer Análisis de Componentes Principales, las siguientes agrupaciones:

\subsubsection{Reagrupación Aderco-Adersur}

Estos dos territorios mantienen entre sí una homogeneidad paisajistica de innegable alcance y continuidad espacial, protagonizada por la dehesa. Podría argumentarse 
en contra de esta propuesta que tal similitud se podría generalizar a buena parte de los terrenos asentados en los suelos desarrollados sobre litologías paleozoicas de la penillanura extremeña. La dehesa del suroeste extremeño, sin embargo, dispone de unas peculiaridades que las diferencian del conjunto: extensión y productividad del encinar y alcornocal, producción herbácea, valor de las montaneras, tradición en la cría del porcino ibérico, tradición en la elaboración de chacinas y embutidos, etc., y todo ello en un área que se extiende desde la frontera con Portugal hasta los muncipios meridionales de la provincia de Badajoz.

La continuidad espacial, la homogeneidad paisajistica y el hecho de que este territorio participe de unas tradiciones comunes en el ámbito ganadero, permiten articular una propuesta que podría redundar en favor de una mayor eficiencia en la aplicación de las políticas de desarrollo rural. Esa es la hipótesis de partida y esa, en definitiva, la razón que nos mueve a tratar de medir el alcance de la reagrupación formulada.

\subsubsection{Reagrupación Zafra-Río Bodion y Tentudía}

Los criterios que han aconsejado la valoración de esta propuesta son similares a los anteriormente expuestos. Cierto es, no obstante, que tanto el GAL de Tentudía como el de Zafra-Río Bodión adolecen de una coherencia física o geógrafica entre sus límites administrativos pero, en cambio, ambos GAL combinan características físico-naturales propias del bosque mediterráneo con otras similares a la campiña de secano. En todo caso, tal circunstancia no constituía un motivo sólido para declinar la posibilidad de realizar la valoración citada, dado que tanto en un Grupo de Acción Local como en el otro hay municipios que podrían formar parte del otro en cuestión, incluso de otros grupos terceros.

\subsection{Propuesta de nueva configuración: Monfragüe}

Esta propuesta de cambio podría sustentarse en el aprovechamiento de las sinergias derivadas de un espacio natural protegido de la entidad del Parque Nacional de Monfragüe, y del tratamiento económico y financiero que afecta su Área de Influencia Socioeconómica.

Los municipios que integran el Área de Influencia Socioeconómica del Parque Nacional de Monfragüe (14) se encuentran actualmente distribuidos en un total de cuatro GAL, una situación ésta que parece poco apropiada para aprovechar esas sinergias y a la que cabe añadir otra contradicción más, y es que el Grupo de Acción Local que utiliza el vocablo "Monfragüe" en su denominación (Ademe), tiene la sede de su GAL en Cañaveral, municipio que no está integrado en el Parque ni en su área de influencia (en la configuración actual 2014-2020 elaborada por la Junta de Extremadura la sede se traslada a Malpartida de Plasencia pero Cañaveral sigue formando parte de dicho GAL). 
Entendemos que la propia figura del Parque Nacional de Monfragüe es de sobra suficiente para constituir el eje vertebrador de una estrategia de desarrollo que dé cohesión a todo el territorio integrado por el Área de Influencia Socioeconómica y que aproveche las sinergias de su reconocimiento nacional e internacional, la afluencia de turistas, la creación de empresas de servicios al turismo, alojamiento, restauración, etc., con independencia de otras actividades artesanales, industriales y comerciales que puedan girar en torno a este eje y que puedan beneficiarse del marchamo del parque. Mantener la situación actual dificultaría el aprovechamiento de estas ventajas, podría suponer una dispersión de esfuerzos y una pérdida de energías que redundarían en perjuicio de las posibilidades que tiene este territorio para vencer algunas de las dificultades extremas que padece tanto en el plano económico como en el demográfico, donde se diseña un cuadro en el que destaca la existencia de una densidad demográfica de 8,2 habitantes $/ \mathrm{Km}^{2}$ y una población de tan sólo 9.622 habitantes con sólo un 12,2\% de niños menores de 15 años y con un jíndice de Envejecimiento! del 205,7\%.

\section{Valoración de las propuestas}

Teniendo en cuenta las propuestas formuladas, y con el fin de valorar su posible impacto en el marco general en que se inscriben cada uno de los Grupos de Acción Local en su configuración actual, se realizó, como ya se indicó con anterioridad, un segundo PCA que dio los siguientes resultados.

En primer lugar, la varianza explicada solamente con los dos primeros componentes alcanza algo más del $72,07 \%$, resultados por tanto muy satisfactorios.

Tabla 6. Varianza explicada y acumulada por componentes

\begin{tabular}{|c|c|c|}
\hline Componentes Principales & \% Varianza Explicada & \% Varianza Acumulada \\
\hline 1 & 59,68 & 59,68 \\
2 & 12,39 & 72,07 \\
3 & 9,51 & 81,58 \\
4 & 5,16 & 86,74 \\
5 & 4,13 & 90,87 \\
6 & 3,07 & 93,94 \\
7 & 2,08 & 96,03 \\
\hline
\end{tabular}

Fuente: Elaboración propia. 
Tabla 7. Cargas factoriales por variables y componentes principales

\begin{tabular}{|l|c|c|c|}
\hline Variables & $P C-1$ & $P C-2$ & $P C-3$ \\
\hline Población joven (menores de 15 años) & 0,26516 & $-0,11543$ & 0,05880 \\
Población adulta (entre 15 y 64 años) & 0,25991 & $-0,12481$ & 0,00534 \\
Población anciana (mayor de 65 años) & $-0,26679$ & 0,12228 & $-0,02825$ \\
Densidad de población (habitantes/km2) & 0,21179 & 0,13125 & 0,13893 \\
Tasa de Crecimiento Real (2001-2013) & 0,24623 & $-0,17547$ & 0,05476 \\
Relación de Feminidad (49 años) & 0,20238 & 0,02646 & $-0,20254$ \\
Índice de Infancia & 0,26526 & $-0,11476$ & 0,05946 \\
Tasa de Maternidad & 0,25346 & $-0,03295$ & 0,09718 \\
Tasa Bruta de Natalidad & 0,26896 & $-0,01875$ & 0,06849 \\
Tasa Bruta de Mortalidad & $-0,24034$ & 0,08641 & $-0,12594$ \\
Índice de Envejecimiento & $-0,25752$ & 0,14106 & $-0,03678$ \\
№ de medio de habitantes por municipio & 0,24855 & 0,13153 & $-0,04864$ \\
Tasa de Dependencia de Mayores & 0,06217 & 0,32608 & $-0,22792$ \\
TRPPA & 0,21945 & $-0,03041$ & $-0,11864$ \\
Afiliados a la S.S., 2013 (Sector Agrario) & $-0,02541$ & 0,16016 & 0,65336 \\
Afiliados a la S.S., 2013 (Sector Industrial) & 0,04705 & $-0,49662$ & $-0,19378$ \\
Afiliados a la S.S., 2013 (Sector Construcción) & $-0,15591$ & $-0,29530$ & $-0,06659$ \\
Afiliados a la S.S., 2013 (Sector Servicios) & 0,07263 & 0,22786 & $-0,59221$ \\
Índice de Actividades Económicas & 0,16463 & $-0,23114$ & $-0,09230$ \\
Tierras Labradas & 0,18378 & 0,39463 & $-0,00755$ \\
Tierras No Labradas & $-0,20545$ & $-0,33619$ & $-0,02783$ \\
Población absoluta (2013) & 0,25330 & 0,08555 & $-0,01378$ \\
\hline
\end{tabular}

Fuente: Elaboración propia.

En el gráfico de cargas factoriales (Gráfico 3), podemos observar la relación existente entre las variables. Como señalamos en el primer PCA, aquellas que están próximas mantienen entre sí elevadas correlaciones positivas, en tanto que las más alejadas tienen correlaciones negativas. 
El primer componente retiene casi el $60 \%$ de la varianza total. Con alta carga factorial positiva, y situadas muy próximas, lo que indica su elevada correlación y que vienen a medir lo mismo, tenemos las variables siguientes:

$X_{1}$ : Población joven (menores de 15 años)

$X_{2}:$ Población adulta (entre 15 y 64 años)

$X_{4}$ : Densidad de población (habitantes $/ \mathrm{km}^{2}$ )

$X_{5}$ : Tasa de Crecimiento Real (2001-2013)

$X_{6}:$ Relación de Feminidad (49 años)

$X_{7}$ : Indice de Infancia

$X_{8}:$ Tasa de Maternidad

$X_{9}$ : Tasa Bruta de Natalidad

$X_{12}$ : No de medio de habitantes por municipio

$X_{14}:$ TRPPA

$X_{20}$ : Tierras Labradas

$X_{22}$ : Población absoluta (2013)

Próximas a este grupo, pero con menor carga, podemos observar en el plano positivo las variables:

$X_{13}:$ Tasa de Dependencia de Mayores

$X_{18}$ : Afiliados a la Seguridad Social (Sector Servicios)

Y, también próximas, pero en el plano negativo la variable:

$X_{19}:$ Índice de Actividades Económicas (2009)

Por otra parte, con alta carga factorial negativa se encuentran las variables:

$X_{3}$ : Población anciana (mayor de 65 años)

$X_{10}:$ Tasa Bruta de Mortalidad

$X_{11}:$ Indice de Envejecimiento

$X_{17}$ : Afiliados a la Seguridad Social, 2013 (Sector Construcción)

$X_{21}$ : Tierras No Labradas

Por lo tanto, este primer componente separa y define perfectamente las variables relacionadas básicamente con la demografia, exactamente igual que en el primer PCA.

El segundo componente retiene el 12,39\% de la variación inicial. Separa con elevada carga positiva (parte superior del Gráfico 3), la variable $X_{15}$ : Afiliados a la Seguridad So- 
cial (Sector Agrario), en contraposición a la variable $X_{16}$ : Afiliados a la Seguridad Social (Sector Industria), con elevada carga negativa (parte inferior del Gráfico 3). Estas dos variables son indicadoras del ámbito socioeconómico pero, en este caso, separa y define muy bien la tendencia agraria o industrial de los Grupos de Acción Local.

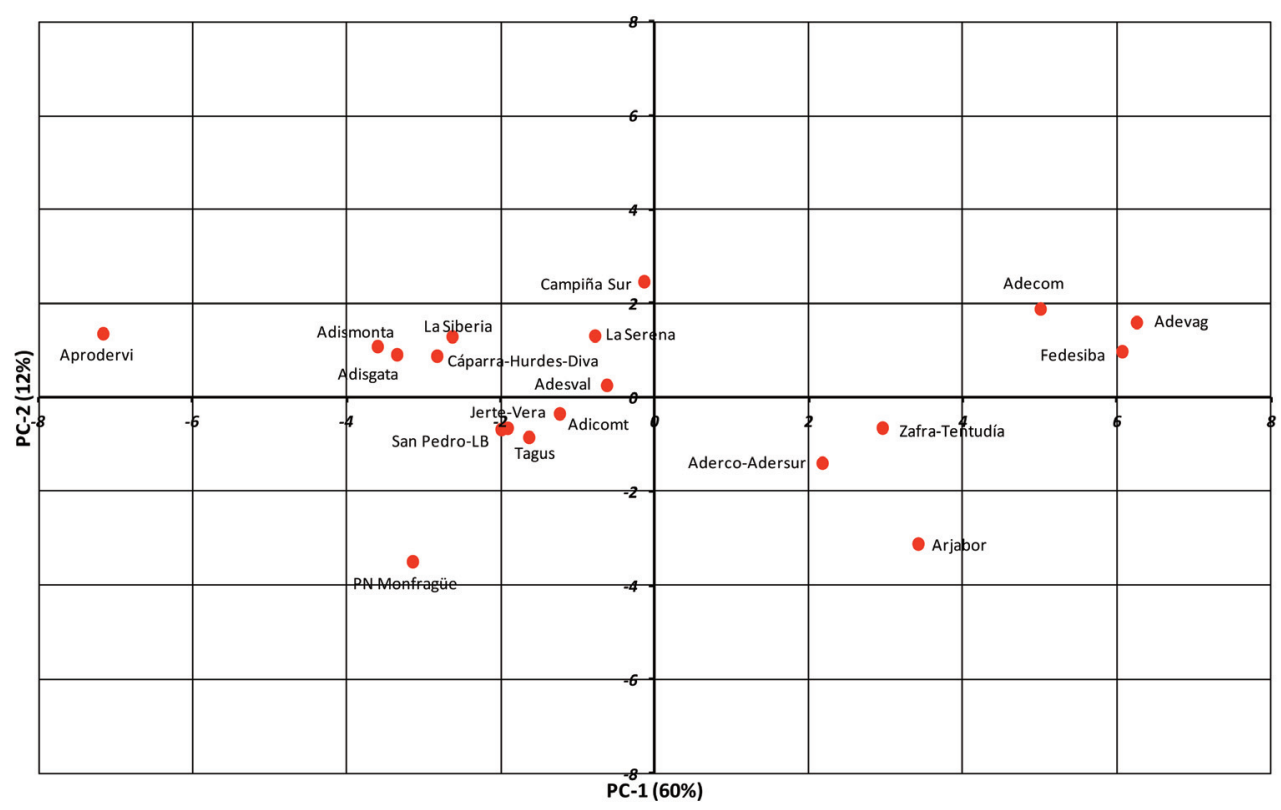

Gráfico 3. Puntuaciones factoriales de los casos.

Fuente: Elaboración propia.

La situación de los Grupos de Acción Local en el espacio relativo definido por los dos primeros componentes podemos observarla en el Gráfico 3 de Puntuaciones Factoriales.

Básicamente, la situación de los Grupos de Acción Local situados en los extremos de ambos componentes son los que presentan un conjunto de características muy representativas de las variables indicadoras del Gráfico 2, en tanto que las que están más hacia el centro, tienen unas características mixtas y/o menos acusadas.

Por lo tanto, con alta puntuación positiva volvemos a encontrar en el primer componente ("Grupos demográficamente progresivos"), los Grupos de Acción Local de Adecom-Lácara, Fedesiba y Adevag. No muy lejos estaría el escenario planteado del caso "Zafra-Tentudía" que, salvo un pequeño cambio de situación en el plano demográfico de Tentudía, no plantea mejoras a la situación actual de ambos Grupos de Acción Local por separado, luego en este caso no parece conveniente el cambio planteado. 
Próximo también se encuentra el caso "Aderco-Adersur" pero, como en el caso anterior, no parece plantear grandes mejoras ni en el plano demográfico ni en el económico, por tanto tampoco en este caso es conveniente llevar a cabo el cambio planteado.

En cambio, los cambios planteados en la configuración territorial de los casos "CáparraDiva-Hurdes" y "Soprodevaje-Adicover" mejora sustancialmente en el plano demográfico aproximándose a los valores centrales de dicho plano y alejándose, por tanto, de las puntuaciones negativas del primer componente que caracteriza a los "Grupos demográficamente más regresivos" aunque, en todo caso, estarían muy próximos a la variable $X_{15^{\circ}} A f i$ liados a la Seguridad Social (Sector Agrario) con carga positiva en el segundo componente.

En el plano negativo más extremo del primer componente seguimos encontrando Aprodervi. Y, por último, el caso del Área de Influencia Socioeconómica del Parque Nacional de Monfragüe que, como decíamos anteriormente, es ciertamente especial y aunque en el plano de ambos componentes no sufra modificaciones importantes, sin embargo creemos que su constitución como GAL puede representar un halo de esperanza para el desarrollo territorial de esta zona tan emblemática del bosque mediterráneo mundial.

Por último, en el Gráfico 4 se puede observar como quedaría la localización de los nuevos casos en el plano factorial de los dos componentes.

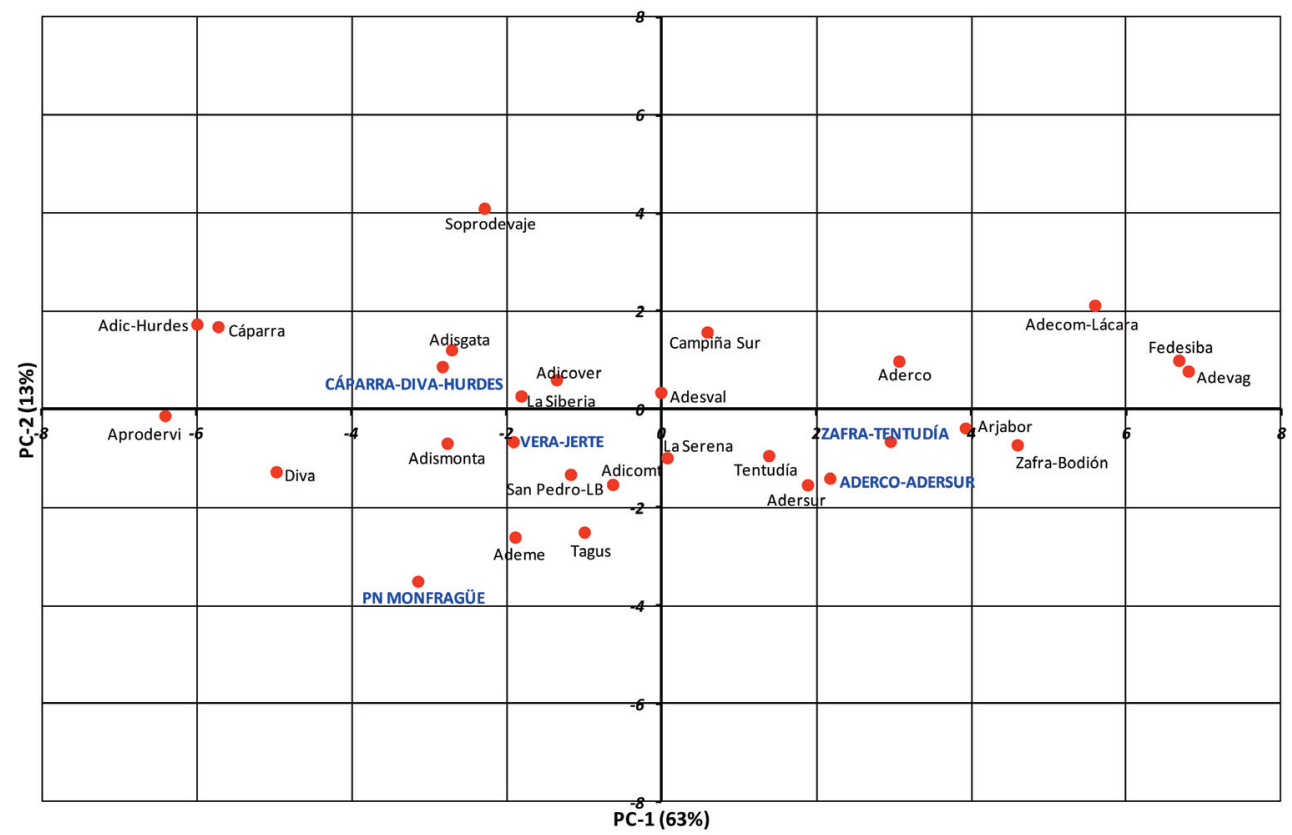

Gráfico 4. Puntuaciones factoriales de los casos.

Fuente: Elaboración propia. 


\section{Conclusiones}

La baja densidad demográfica de Extremadura, la dispersión geográfica de la población, el elevado grado de envejecimiento demográfico, el creciente fenómeno de la masculinización, la persistencia de saldos migratorios negativos, los desequilibrios estructurales de la economía, con una fuerte dependencia del sector agrario y el escaso desarrollo de la industria y los servicios, convierten a la región en un interesante laboratorio para valorar el impacto de las políticas europeas de Desarrollo Rural sobre el territorio.

La primera evidencia que se presenta al analizar los resultados obtenidos durante el período de programación 2007-2013, es la existencia de diferencias sensibles en la distribución de los fondos, el presupuesto ejecutado, las iniciativas puestas en marcha, el volumen de empresas creadas, el empleo generado o el peso de la iniciativa privada en el conjunto de proyectos gestionados. Se ha destacado, de este modo, que no existe una distribución lógica de la subvención pública entre los distintos Grupos de Acción Local, de modo que este parámetro experimenta una disminución a media que aumenta, por ejemplo, el tamaño poblacional del Grupo o su extensión superficial.

Por otro lado, los Grupos de Acción Local con mayor dimensión poblacional consiguen tener unos porcentajes más altos de inversiones privadas que los de menor entidad, lo que evidencia una diferente capacidad para dinamizar el territorio a través de los recursos humanos y financieros locales y, por ende, una mayor dependencia de la inversión pública.

En lo que respecta a los gastos de funcionamiento de los Grupos, se advierte que estos aumentan a media que disminuye su tamaño poblacional y territorial, lo que en principio podría resultar contradictorio por cuenta que parecería lógico que el equipo técnico y los gastos de gestión dependieran, en parte, de dichos parámetros.

Finalmente, la distribución de los gastos por medidas y submedidas es totalmente distinta en cada Grupo de Acción Local, con independencia de que entre algunos de ellos existan similares recursos y potencialidades lo que, evidentemente, podría haber propiciado la coincidencia en sus estrategias de desarrollo.

En la mayor parte de los casos, esta diversidad encuentra explicación en los contrastes territoriales de la propia región y, en consecuencia, en las diferentes posibilidades de desarrollo que ofrecen los territorios: recursos naturales, humanos y económicos; situación geográfica respecto de los centros urbanos regionales y extrarregionales; infraestructuras de comunicación y transportes, etc. No obstante, son diversas las ocasiones en que la diversidad de los resultados no se explica por la existencia manifiesta de diferencias territoriales.

La realización de un primer análisis de Componentes Principales permite colegir que la situación socioeconómica y demográfica de los GAL están directamente relacionadas 
con el tamaño poblacional, de modo que los GAL más regresivos demográficamente y más débiles desde el punto de vista económico son aquellos que cuentan con menor tamaño poblacional, esto es: Adic-Hurdes (6.190 habitantes), Aprodervi (13.937 habitantes), Cáparra (9.267 habitantes), Diva (7.979 habitantes), Soprodevaje (11.267 habitantes) o Ademe (9.622 habitantes), como casos más extremos. En cambio, aquellos más dinámicos demográfica y económicamente vienen a coincidir con los GAL más poblados: Adevag (89.087 habitantes), Fedesiba (86.121 habitantes), Adecom-Lácara (62.616 habitantes), Zafra-Río Bodión (47.423 habitantes) o Arjabor (38.366 habitantes), entre otros.

Partiendo de estas premisas, se ha procedido a plantear una primera propuesta basada en la posibilidad de pretender mayores niveles de eficiencia y eficacia en la implementación de la metodología LEADER tratando de configurar grupos con mayor masa poblacional crítica. En esta propuesta se han incluido la reagrupación de los GAL de Cáparra, Diva y Hurdes y la unión de Soprodevaje y Adicover.

Una segunda propuesta ha pretendido un mejor posicionamiento de algunos Grupos atendiendo a agrupaciones que mantengan entre sí una sustantiva homogeneidad territorial. En este caso, se ha planteado la posibidad de unificar los Grupos de Aderco y Adersur, así como los de Zafra-Río Bodión y Tentudía.

Por último, se ha planteado la posibilidad de reconfigurar el actual Grupo de Ademe, obteniendo una nueva estructura territorial que agruparía los municipios integrados en el Área de Influencia Socioeconómica del Parque Nacional de Monfragüe (Mapa 2).

Para valorar la idoneidad de estas propuestas, se procedió a una nueva aplicación del Análisis de Componentes Principales con la introducción de las agrupaciones anteriormente citadas, con unos resultados que conducen a valorar muy positivamente los correspondientes a la unión de los GAL de Adic-Hurdes, Diva y Cáparra; Soprodevaje y Adicover, y la nueva configuración como GAL del Área de Influencia Socioeconómica de Monfragüe.

En virtud de estas consideraciones, debe destacarse las posibilidades que ofrece el análisis factorial para valorar el grado de eficacia y de eficiencia con que se aplican los fondos comunitarios que gestionan los Grupos de Acción Local para contribuir al desarrollo de los territorios rurales, valoración esta que, en nuestro caso, permite sugerir reajustes territoriales que superen los obstáculos y rémoras que plantean los criterios subjetivos para lograr unos resultados óptimos en la gestión, aplicación y seguimiento de las inversiones realizadas. 


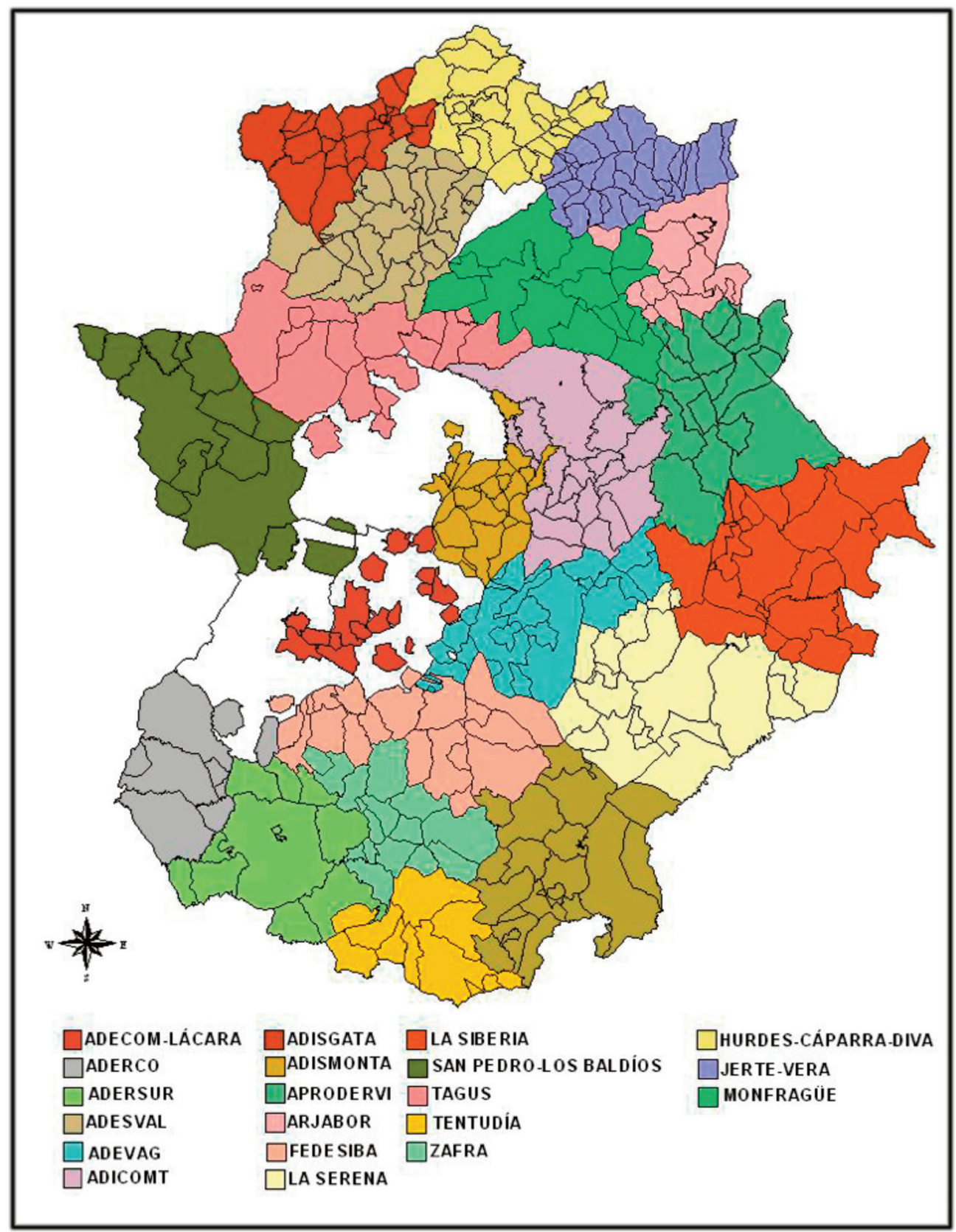

Mapa 2. Grupos de Acción Local de Extremadura. Propuesta de configuración. Fuente: Elaboración propia. 


\section{Bibliografía}

Aguilar, E., 2013. Análisis factorial para el estudio de la mortalidad de Costa Rica. Período 19002010. Población y Salud en Mesoamérica. Vol. 10, núm. 2, pp. 1-25.

Aguilera, P., Garrido, A., 2001. Aplicaciones ambientales del análisis multivariante. Universidad de Almería. Servicio de Publicaciones.

Aguilera, M.J., González, M.P., 1988. La diferenciación espacial del envejecimiento demográfico de la Comunidad Autónoma de Madrid. Revista de la Facultad de Geografía e Historia, 3, pp. 73-106.

Altuzarra, A., 2010. Identificación de submercados de vivienda en España. Revista de métodos cuantitativos para la economía y la empresa, 10, pp. 19-42.

Arias, P., Alarcón, S. y Botey, M., 2016. La caracterización a través del análisis factorial de las empresas agroalimentarias según sus obstáculos a la innovación. Economía Industrial, 400, pp. 139-149.

Ávila, R., 1993. Nueva perspectiva de las migraciones interiores españolas. Anales de Geografía de la Universidad Complutense, 113, pp. 111-126.

Biotto, G., Silvestri, S., Gobbo, L., Furlan, E., Valenti S. y Rosselli, R. 2009. GIS, multi criteria and multi factor spatial analysis for the probability assessment of the existence of illegal landfills, International Journal of Geographical Information Science, 10, pp. 1233-1244.

Callizo, J. y Lacosta, A.J., 1997. La explicación teórica de la potencialidad turística del medio natural. Verificación en Aragón del modelo de Warszynska a partir del análisis factorial. Geographicalia, 35, pp. 19-28.

Cejudo, E., Navarro, F., 2009. La inversión en los programas de desarrollo rural. Su reparto territorial en la provincia de Granada. Anales de Geografia de la Universidad Complutense, 29, pp. 37-64.

Cuadras, C., 1981. Métodos de Análisis Multivariante. Barcelona: Eunibar.

Cuadras, C., 2011. Nuevos métodos de análisis multivariante. Barcelona: CMC Editions.

Chueca, J., 1991. Aplicación de métodos de análisis factorial en el establecimiento de una tipología de los glaciares rocosos del Pirineo Central Oscense. Cuaternario y Geomorfología, 5 , pp. $27-43$.

Chuvieco, E., 1996. Fundamentos de teledetección espacial. Madrid: Ed. RIALP.

De Esteban, G., 2000. Actitudes de los españoles ante los problemas ambientales. Observatorio Medioambiental, 3, pp. 107-122.

Díaz, P., 1996. La diferenciación socio-espacial en la ciudad de Burgos durante los años de la posguerra. Espacio, Tiempo y Forma, Serie VI, Geografía, vol. 9, pp. 103-131.

Emma, N., Cambillo, M., Ysela, D., Agüero, P., Dora, F. y Rafael, H., 2014. Análisis factorial múltiple y metodología STATIS en el análisis de la transición demográfica en el Perú. PESQUIMAT, vol. XVI, 1, pp. 64-72.

Esparcia, J., Escribano, J., 2012. La dimensión territorial en la programación comunitaria y el nuevo marco de políticas públicas: desarrollo rural, reforma de la PAC y nuevo LEADER. Anales de Geografia, vol. 32, 2, pp. 227-252. 
Fernández, M., 2009. Bolivianos en España. Revista de Indias, 245, pp. 171-198.

García, P., Pérez, M.E., García, J.M., Redondo M.M., Sanz, J.J. y Navarro, A., 2014. Sellado de suelos a partir de teledetección y SIG: Estudio en el Tajo Medio-Alto. Dpto. de Análisis Geográfico Regional y Geografía Física. Universidad Complutense de Madrid.

Gorsevskia, P., Donevska, K., Mitrovskic, D. y Frizado, P., 2012. Integrating multi-criteria evaluation techniques with geographic information systems for landfill site selection: A case study using ordered weighted average. Waste Management, 2, pp. 287-296.

Jiménez, B.C., 1984. Aproximación metodológica al estudio de la diferenciación residencial urbana en Madrid. Anales de Geografía de la Universidad Complutense, 4, pp. 167-187.

Jordá, R. y Ruíz, F., 2009. La internacionalización de las empresas innovadoras andaluzas. Mecanismos, evolución, fases y variables clave", Cuadernos de Geografía, 85, pp. 23-54.

Jordá, R., Ruíz, F. y Lucendo, A.L., 2013. Factores territoriales de localización y caracterización de los vertederos incontrolados en Andalucía. Scripta Nova, p. 435.

Leco, F., Pérez, A. y Mateos, A.B. (2017): Crisis demográfica en la Extremadura rurak: valoración a través de los Grupos de Acción Local (2007-2014). Cuadernos Geográficos, 56(1), pp. 76-100.

Lora, G. y Aparicio, F., 1991. Delimitación de áreas sociales en el Barrio de la Concepción. Un ensayo de ecología factorial a escala microespacial. Espacio, Tiempo y Forma, Serie VI, Geografia, vol. IV, pp. 189-216.

Mallo, F., 1985. Análisis de componentes principales y técnicas factoriales relacionadas. León: Editorial Universidad de León.

Marquez, D., Galindo, L., García, A. y Foronda, C., 2005. Eficacia y eficiencia de Leader II en Andalucía: aproximación a un índice-resultado en materia de turismo rural. Geographicalia, 47, pp. 137-152.

Méndez, R., Michelini, J.J., Romeiro, P. y Sánchez, S., 2006. Ciudades intermedias y desarrollo territorial en Castilla-La Mancha. Xeográfica, 6, pp. 69-93.

Montoya, O., 2007. Aplicación del análisis factorial a la investigación de mercados. Caso de estudio. Scientia et Technica, 35, pp. 281-286.

Nieto, A.y Cárdenas, G., 2016. El método LEADER en Extremadura en los últimos 25 años. En Ruíz, A.R.-Serrano, M.A.y Plaza, J. (eds): Treinta años de Política Agraria Común en España. Agricultura y multifuncionalidad en el contexto de la nueva ruralidad. Ciudad Real: Óptima Diseño e Impresión.

Nieto, A. y Cárdenas, G., 2015. Los Grupos de Acción Local en el período de programación FEADER (2007-2013) en Extremadura. Revista de Estudios Extremeños, 1, pp. 605-638.

Ocaña, C. y Navarro, S., 2001. Edad y movilidad migratoria en Andalucía, nuevos patrones territoriales. Baética: Estudios de Arte, Geografía e Historia, 23, pp. 171-191.

Pearson, K., 1901. On lines and planes of closest fit tosystems of point in space. Philosophical Magazine, 6, pp. 559-572.

Pérez, C., 1990. Tipología de los asentamientos rurales en la Comunidad de Madrid. Espacio, Tiempo y Forma, Serie VI, Geografía, vol. 3, pp. 93-112. 
Redondo, J.M., 1988. Evaluación del impacto ambiental de los depósitos de estéril en la minería del carbón según el índice Q. Cuneca de Ciñera-Matallana (Sector oriental), León. Eria, pp. 251-258.

Rúa, A., Redondo, R. y Del Campo, C., 2003. Distribución municipal de la realidad socioeconómica gallega. Revista Gallega de Economía, vol. 2, pp. 1-20.

Saco, A., 1995. Dinamismo sociodemográfico y ruralidad en Galicia. Agricultura y sociedad, 76, pp. $187-202$.

Sánchez, D., 2009. Geografía del envejecimiento vulnerable y su contexto ambiental en la ciudad de Granada: discapacidad, dependencia y exclusión social. Cuadernos Geográficos, 45, pp. 107-135.

Sánchez, J.M., 1998. Tipología turística municipal de Extremadura basada en el análisis factorial de componentes principales. Lurralde: Investigación y Espacio, 21, pp. 95-119.

Santos, J.M., 1991. La técnica factorial y su aplicación en el campo geográfico. Espacio, Tiempo y Forma, pp. 13-78. Madrid: Universidad Nacional de Educación a Distancia.

Santos, J.M., 1998. La diferenciación de áreas de mercado laboral juvenil homogéneas, en el municipio de Getafe. Espacio, Tiempo y Forma, Serie VI, Geografía, vol. 11, pp. 145-155.

Seoane, G., Rodríguez, S. y Arce, C., 2000. Comparación de modelos de evaluación del servicio de autobús urbano para usuarios reales y potenciales. Psicothema, vol. 12, supl. no 2, pp. 522-525.

Serra, P., Tulla, A.F., Vera, A., Pallarés, M. y Badia, A., 2006. Dinámicas de los municipios catalanes en los últimos 20 años a través del análisis factorial. En Camacho, M.T., Cañete, J.A. y Lara, J.J. (eds). El acceso a la información espacial y las nuevas tecnologías geográficas, pp. 481-490. Granada: Universidad de Granada.

VV.AA., 2014. Treinta años de Economía y Sociedad Extremeña, 1983-2013. Badajoz: Excma. Diputación Provincial.

Vidal, E., Gil, F. y Domingo, A., 2008. La distribución territorial de la población femenina extracomunitaria en España: factores demográficos y laborales. Papeles de Geografía, 47-48, pp. $193-213$. 